\title{
Perception et interprétation des émotions dans le débat radiodiffusé par les étudiants avancés de philologie romane
}

\author{
The reception and construal of emotions in talk radio \\ programmes by advanced students of Romance philology
}

\author{
Bernadeta Wojciechowska \\ Université Adam Mickiewicz \\ bewoj@amu.edu.pl
}

\begin{abstract}
The article focuses on the French philology students' skills of interpreting emotions in radio debates. Data analysis was conducted with the use of the interactional approach, discourse analysis and the rhetorical argumentation approach. The aim was to better understand the students' interpretative actions and especially, their perception of ethos and pathos. The second objective of the study was to conduct a pilot test of the methodology inspired by research on argumentation in discourse in order to analyze persuasive discourse in a foreign language.
\end{abstract}

Keywords: listening comprehension in a foreign language, media debate, critical interpretation, rhetoric strategies, emotions

\section{INTRODUCTION}

Les compétences orales en langues étrangères visées au niveau avancé (B2-C2, selon le Cadre européen commun de référence pour les langues, 2001) dépassent largement les capacités et les connaissances nécessaires pour réaliser des objectifs courants de la vie quotidienne, aussi bien en réception qu'en production. Autant les niveaux de compétences plus bas (jusqu'à B1) se réfèrent à des situations sociales fortement conventionnelles restant en rapport avec le monde des objets disponibles dans l'environnement immédiat, qu’il soit réel ou fictif, de l'utilisateur/apprenant, autant les compétences de haut niveau sollicitent 
beaucoup plus les opérations intellectuelles d'analyse et de synthèse agissant sur les concepts abstraits. Ce travail intellectuel sur les concepts élaborés à l'origine majoritairement en langue maternelle et leur confrontation avec les visions du monde circulant dans une culture étrangère ${ }^{1}$ apparaît comme l'un des principaux enjeux d'enseignement/apprentissage. Sa valeur réside dans la jonction de la formation intellectuelle et du développement langagier, promettant une réelle autonomie communicative du sujet. Cet objectif concorde avec la finalité de l'enseignement/apprentissage des langues étrangères présentée dans le Cadre européen commun (2001) qui est de former un apprenant acteur social de plein droit.

Il semble que la réalisation des objectifs ainsi formulés devrait privilégier le discours argumentatif, principal moyen de création de l'opinion publique et fondement du processus décisionnel dans les sociétés démocratiques ${ }^{2}$. Il faudrait donc garantir à l'apprenant un large accès au débat public mais aussi lui fournir des outils intellectuels adéquats pour les comprendre et interpréter, et donc développer une attitude critique, y compris par rapport aux émotions véhiculées et suscitées par les débats. Autant ce premier postulat ne pose pas de problème à l'ère de la télévision, des podcasts et autres technologies d'Internet, autant le deuxième risque d'être difficile à atteindre, vu le ralentissement de la "lecture" qu'il impose dans un monde à grande vitesse. C'est une raison de plus pour y donner toute sa place dans le contexte des études philologiques.

Les premières recherches faites auprès des étudiants avancés $(\mathrm{B} 2 / \mathrm{C} 1)$ de philologie romane (p.ex. Górecka et Wojciechowska, 2013) à l'Université Adam Mickiewicz de Poznań ont permis de mettre à jour les difficultés des apprenants adultes à interpréter de façon critique le discours argumentatif. Ceci se manifestait essentiellement par une tendance fort répandue à se limiter au repérage et à la restitution des idées explicites du texte. Notons au passage qu'une telle attitude des apprenants découle essentiellement des habitudes prises à des étapes antérieures où on travaille l'écoute à travers la reformulation ou, éventuellement, la réfutation sans aucune analyse critique (voir aussi Allen, 2012, Lafontaine, 2004).

La présente étude s'inscrit dans la lignée de ces travaux, elle vise à examiner comment les étudiants perçoivent les émotions dans un débat radiodiffusé et comment ils en tiennent compte dans l'accomplissement d'une tâche interprétative. L'originalité de cette étude tient entre autres à l'élargissement du cadre théorique qui tente d'intégrer l'approche discursive de l'écoute en langue

\footnotetext{
${ }^{1}$ Dans différentes communautés constitutives de cette culture, véhiculées par des individus.

${ }^{2}$ Le lien entre la qualité des médias et la démocratie est un sujet qui n'a pas besoin d'être développé tellement il est présent dans la réflexion contemporaine. L’importance des compétences rhétoriques pour la société démocratique a été mise en relief p.ex. par Ph. Breton (2007).
} 
étrangère (Górecka, Wilczyńska, Wojciechowska, sous presse) à l'approche rhétorique de l'argumentation dans le respect des contraintes cognitives propres à la situtation d'écoute et du traitement (accès limité aux représentations sociales, gestion des ressources attentionnelles, limite de la mémoire de travail) du discours oral en langue étrangère.

\section{CONDITIONS COGNITIVES DE L'ACTIVITÉ INTERPRÉTATIVE}

L'activité interprétative suscite de plus en plus d'intérêt au point que l'on parle du "tournant interprétatif" dans les sciences humaines (Vandendorpe, 2001: 81). Tout porte à croire que le même tournant se réalise, à une échelle plus individuelle, pour les étudiants mis devant une tâche d'analyse de débats. Alors qu'ils étaient focalisés jusque là sur ce qui est «dit» et éventuellement « comment c'est dit », ils sont obligés de répondre aux questions de type « pourquoi on dit ce qu'on dit» et "pourquoi on le dit de cette façon là », et donc ayant pour but de saisir l'orientation argumentative du discours (Charaudeau, 2008).

Au niveau B2/C1, la saisie du contenu ne pose pas vraiment de problème, elle se réalise par une compréhension presque automatique, immédiate, résultant de la mise en correspondance relativement rapide d'une donnée présente dans le discours à un contexte évoqué par ce discours. Contrairement à la compréhension ainsi conçue, l'interprétation demanderait un effort cognitif supplémentaire et sous-entendrait une capacité de distanciation par rapport au sens construit dans un premier temps (Vandendorpe, 2001), indispensable pour déjouer les mécanismes de mise en scène discursive (Grize, 2004, Vignaux, 2004), pour l'évaluer, voire contester. Cela pourrait faire croire que l'interprétation est toujours secondaire à la compréhension, mais Ch. Vandendorpe (2001) démontre qu'une fois l'interprétation faite, elle constitue une base pour la compréhension de la suite du discours. Ce mouvement de va-et-vient entre compréhension et interprétation est décrit par les modèles interactifs de construction du sens dans lesquels les données de bas niveau sont rapportées à des données souvent hypothétiques de haut niveau et parallèlement, ces hypothèses servent de stratégies qui orientent le recueil de données de bas niveau.

Dans nos travaux précédents, nous avons défendu l'approche discursive de l'oral en insistant sur la notion du genre conçu comme une pratique sociale qui constitue une sorte de cadre global de l'activité langagière propre à un domaine de la vie, avec sa fonctionnalité propre, ses rôles et identités, ses thèmes de prédilection, ses schémas interactifs propres (monologaux, dialogaux), son style (caractéristiques textuelles et formelles). Une telle approche signifie que les 
compétences interprétatives de l'apprenant devraient lui permettre d'intégrer les données de différents niveaux en rapport avec la situation de communication. Dans la perspective pédagogique de l'enseignement/apprentissage de la compréhension du débat polémique, on peut se poser la question de savoir si on peut distinguer une ou quelques caractéristiques capitales pour l'interprétation, suffisamment saillantes pour orienter l'analyse de la polémique en cours. Par cet aspect, on peut admettre que ce n'est pas la valeur logique de l'argument ni même une manière idéale de le construire qui décident du succès de persuasion parce que, comme l'ont montré de nombreuses analyses, la polémique se réalise à travers différents types de textes (Amossy, 2011/12, Branca-Rosoff, 2007, Charaudeau, 2008). Parfois même, le non respect du schéma formel dans le discours persuasif fait, comme le remarquent à juste titre D. Vincent et G. Bernard Barbeau (2012: 2) que « les actes indirects et les implicites sont au moins aussi redoutables que les raisonnements formels ». Plus encore, on peut penser que cet attachement à la structure formelle de l'argumentation et sa rationalité (logos), est illusoire ${ }^{3}$ (Ducrot, 2004) et contribue dans une certaine mesure à la difficulté de se distancier et d'apporter un regard critique sur l'ensemble des démarches de persuasion, voire à sous-estimer celles qui portent sur les sentiments (ethos et pathos).

\section{EXPLOITATION DIDACTIQUE DE LA DIMENSION RHÉTORIQUE DU DÉBAT}

À la lumière des réflexions faites ci-dessus, on posera ici comme hypothèse que pour ce qui est du genre débat polémique, la caractéristique essentielle pour l'activité interprétative pourrait être celle de la visée de persuasion, et donc sa dimension rhétorique. La priorité donnée à la dimension rhétorique n'exclut pas les dimensions textuelles, interactives et dialogiques mais soumet en quelque sorte leur perception et analyse aux enjeux de persuasion en constituant ainsi une sorte de macrostratégie interprétative. En effet, le recours aux arguments rationnels (logos) ${ }^{4}$ est inséparable dans le débat polémique d'autres formes d'influence (pathos, ethos), puisque persuader consiste à toucher l'auditeur de

\footnotetext{
${ }^{3}$ En analysant les enchaînements en "donc", O. Ducrot conclut qu'ils « relèvent autant du coup de force que les brutales affirmations. Leur efficacité persuasive [...] relève avant tout de l'effet qu'ils ont sur l'ethos » (Ducrot, 2004 : 32).

${ }^{4}$ O. Ducrot soutient que les enchaînements argumentatifs utilisés dans le cadre d'une stratégie polémique servent à donner une bonne image de l'orateur. Par exemple, en parlant de la concession, il évoque parmi ses fonctions celle d'améliorer l'ethos «l'orateur a l'air d'un homme sérieux, donc fiable, puisque, avant de choisir sa position $\mathrm{Z}$, il a aussi fait attention aux objections possibles contre Z » (Ducrot, 2004 : 29).
} 
manière à le faire croire, à le séduire, cela au détriment de l'opposant. Ce parti pris revient à valoriser, suite aux adeptes de l'argumentation dans le discours, l'auditoire (Amossy, 2011/12, Tindale, 2009) car c'est à lui que le locuteur doit s'adapter ${ }^{5}$ et c'est lui qui en est le dernier juge (Doury, 2003).

\section{3. ÉMOTIONS DANS LE DÉBAT. ETHOS ET PATHOS}

Conformément à la réflexion faite ci-dessus, nous nous sommes particulièrement intéressée dans notre recherche à la perception et à l'interprétation des émotions : du pathos lorsqu'il s'agit de toucher, émouvoir l'auditoire et de l'ethos lorsqu'il s'agit pour le locuteur de convaincre l'auditoire de l'expertise et de ses valeurs morales et discréditer l'interlocuteur. Pour ce qui est de ce dernier, $P$. Charaudeau en parle en termes d'enjeu de légitimation « qui vise à déterminer la position d'autorité du sujet» (2008:6). Toujours selon P. Charaudeau, l'autorité du locuteur peut être validée à travers le recours à l'autorité institutionnelle, position qui est l'effet des savoirs ou compétences reconnues du sujet (expert, savant, spécialiste) et/ou qui résulte du pouvoir de décision (responsable d'une organisation). Elle peut se fonder, exclusivement ou complémentairement, sur l'autorité personnelle, et alors elle a trait au pouvoir de séduction du sujet et la mise en avant de ses valeurs morales. Ces différents types d'ethos font partie des représentations sociales, si bien que leur pouvoir d'influence dépend de l'activation des savoirs et valeurs partagés dans une communauté donnée. En plus, les identités discursives des interlocuteurs sont soumises à l'évaluation et à la négociation interactive (Kerbart-Orecchoni, 2005), ce qui se fait, et parfois de manière fort spectaculaire, dans les débats polémiques.

Un autre enjeu, cité par P. Charaudeau (2008) qui nous intéressera ici comme faisant partie de la macrostratégie interprétative est celui de captation. Il s'agit de faire entrer l'auditeur dans le cadre interprétatif du sujet polémiquant et de le détourner de celui proposé par l'interlocuteur. Cela se réalise entre autres, par la mise en place du sentiment de connivence ainsi que par les procédés de dramatisation ayant pour but de faire ressentir certaines émotions.

\section{PRÉSENTATION DE LA RECHERCHE}

La réflexion théorique brièvement esquissée ci-dessus nous a conduit à poser la question sur la manière dont les étudiants qui n'ont pas subi d'entrainement

\footnotetext{
${ }^{5}$ Par ailleurs, la manière dont les interlocuteurs du débat radiophonique ou télévisuel gèrent l'hétérogénéité de leur public en dit long sur leurs intentions et sur les représentations qu'ils s'en font.
} 
rhétorique s'acquittent de la tâche d'évaluation critique d'un passage du débat, et plus exactement, comment ils perçoivent les liens entre l'émotionnel (ethos et pathos) et le rationnel (logos). Nous avons organisé notre examen autour de deux questions suivantes :

1) Sur quelles catégories d'analyse les interprétations fournies par les apprenants se basent-elles?

2) Comment les indices recueillis au niveau discursif et interactif sont-ils rapportés aux visées polémiques (logos, pathos, ethos) ?

\subsection{CONTEXTE ET DÉROULEMENT DE LA RECHERCHE}

Les données pour cette analyse ont été recueillies auprès de 12 étudiantes de philologie romane Master 2 de l'Université Adam Mickiewicz de Poznań à qui on a d'abord demandé d'écouter à la maison, autant de fois qu'elles voulaient, le podcast de la radio France Inter de l'émission "Le téléphone sonne" du mardi 3 décembre 2013 intitulée "Le succès croissant du tatouage" ${ }^{\text {" }}$. Cette écoute était accompagnée de questions préparées par l'enseignant auxquelles les étudiantes devaient répondre. Certaines questions de ce questionnaire ont été ensuite reformulées et proposées à la discussion à deux. Cette discussion était enregistrée par les étudiantes ${ }^{7}$. La discussion était censée aider la prise de distance et la justification des évaluations de chacun. Dans la présente analyse, seront utilisés principalement les fragments retranscrits ${ }^{8}$ de la discussion autour de la question, ici question A, portant sur un passage précis du débat, formulée comme suit : « Comment analysez-vous la stratégie rhétorique de Tin-tin dans la séquence où il compare les risques accompagnant la pratique de tatoutage et les infections mortelles contractées à l'hôpital ? ». Certaines données relatives à ce passage ont été présentes aussi dans la réponse à une autre question, ici question $\mathrm{B}$, plus englobante (la dernière de la liste) : "Comment les émotions des participants au débat se manifestent-elles dans l'émission ? Citez (chacune) au moins trois manifestations explicites et trois manifestations implicites des émotions qui animent les intervenants ? Lesquelles de ces manifestations vous paraissent-elles

\footnotetext{
${ }^{6}$ L'émission en question est disponible sur le site de la radio, à l'adressse suivante : http://www.franceinter.fr/emission-le-telephone-sonne-le-succes-croissant-du-tatouage

${ }^{7}$ La consigne a été la suivante : «après avoir écouté l'émission sur le tatouage, enregistrez une discussion à deux autour des questions fournies par l'enseignant. Avant de vous mettre à discuter avec l'autre, faites une réflexion individuelle en vue de préparer votre propre analyse des questions proposées. Lors de la discussion, confrontez vos points de vue respectifs en vous situant par rapport à l'analyse faite par l'autre ».

${ }^{8}$ Nous les avons reproduits avec les erreurs commises par les apprenants.
} 
justifiées et acceptables et lesquelles non ? » Nous les prenons en compte aussi lorsqu'elles éclairent ou complètent les réponses à la question précédente.

Quoique notre analyse soit essentiellement qualitative, nous l'accompagnons de remarques à caractère fréquentiel. Par ailleurs, nous tenons à préciser que les fragments analysés sont représentatifs pour l'ensemble de la discussion des apprenants sur le débat choisi.

\subsection{DÉBAT POLÉMIQUE SUR LES TATOUAGES}

Avant de passer à la présentation et l'examen des données, disons quelques mots à propos du débat lui-même pour fournir un contexte général aux réponses des étudiantes. A l'origine du débat choisi se trouve un projet de loi interdisant 59 colorants utilisés pour faire des tatouages. Le sujet est introduit par l'animateur Pierre Weill (PW) en termes suivants: "Quels sont les risques de cette pratique? Une soixantaine de colorants devraient être interdits au début de l'année prochaine. Plus généralement, pourquoi cette passion pour le tatouage, notamment chez les jeunes ? ». Ce sujet est débattu par les invités de PW qui sont Marie Cipriani-Crauste (MCC), psychosociologue, ex-chercheur au CNRS, non concernée directement par le projet ; Jacques Bazex (JB), professeur, membre de l'académie de médecine et co-auteur d'un rapport en 2007 de l'Académie nationale de médecine sur les complications de la pratique du tatouage, favorable au projet de loi, Tin-tin (Tt) tatoueur, président du SNAT (syndicat national des artistes tatoueurs) farouchement opposé au projet de loi. Les fragments analysés de la discussion des apprenants portent sur l'intervention de Tin-tin (lignes 4-9) que nous reproduisons ci-dessous entourés d'interventions adjacentes :

1. MCC ... mais le tatouage s'est toujours fait et dans des conditions peut-être pas très encadrées

2. dans le passé mais est-ce que nous avons comme ça des épidémies et des problèmes

3. énormes ?...

4. Tt non il n'y en a pas et aujourd'hui en plus aujourd'hui c'est tellement encadré excusezmoi de

5. de d'intervenir mais c'est tellement encadré aujourd'hui que je pense que les médecins

6. feraient mieux de balayer devant leur porte étant donné qu'il y a deux fois plus de

7. morts d'infections nosocomiales que de morts sur la route [oui] il y a plus de dix mille morts par

8. an [(rire de fond), d'accord, d'accord] qui meurent dans les hôpitaux sous les soins de

9. de des professeurs comme professeur Bazex...

10. PW : :.. je reviens Marie cette attirance de des jeunes pour le tatouage ils sont e comment

11. expliquez-vous cela?

Extrait 06'37', - 06'58', du débat 


\section{ANALYSE DES RÉSULTATS}

\subsection{ORIENTATION POLÉMIQUE. DOMINATION DES CATÉGORIES INTERACTIVES}

L'analyse des discussions des étudiantes montre qu'elles n'ont aucun mal à saisir le caractère polémique du débat et à identifier les deux opposants principaux. Elles le développent essentiellement dans la réponse à la question $\mathrm{B}$ portant sur l'ensemble du débat. L'indice le plus fréquemment évoqué sur lequel elles se fondent pour parler des émotions est celui de la gestion conflictuelle des tours (interruption, longueur des interventions de Tin-tin, autodésignation, interruptions, chevauchements, rythme des échanges). On en voit une illustration dans l'exemple 1 et 2 ci-dessous :

\section{Exemple 1}

N. 1. En général la discussion est vivante et agressive

O. 2. de temps en temps

N. 3. oui lorsqu'il s'agit de Tin-tin qui fait intervenir la parole de

O. 4. ... de monsieur Bazex...

N. 5. ...de monsieur Bazex.

'O. 6. oui et on voit bien qu'ils sont pas d'accord et l'un n'aime pas je sais pas le dermatologue ou le euh les

7. choses

N. 8. il augmente la voix

O. 9. oui oui oui il coupe la parole il coupe la parole très très souvent

N/O.Tatouage.QuestionB, 1-9

Dans les lignes 6-7 de l'exemple 1, les étudiantes identifient parfaitement les sentiments manifestes dans la voix des intervenants « on voit bien qu'ils ne sont pas d'accord et l'un n'aime pas le dermatologue» ou dans la ligne 8 «il augmente la voix ». Un autre exemple de cette identification, cette fois-ci en référence à l'activité du journaliste-animateur est présent dans l'exemple 2 dans les lignes 22-24, et par rapport aux interlocuteurs dans les lignes 26-30.

\section{Exemple 2}

D. 22. Je ne sais pas si tu as fait attention que il y un moment c'est le journaliste l'animateur de cette débat

23. est un peu irrité c'est le moment où il essaie de calmer monsieur Tin-tin laissez parler monsieur

24. Bazex oui et je pense que ici on voit bien aussi les émotions de de journaliste

R. 25. C'est ça

D 26. Et je pense aussi que la personne de Tin-tin est pleine d'émotions négatives parce qu'il attaque

27. presque tout le temps monsieur Bazex il essaie de montrer le manque de savoir manque de raison 
28. c'était l'exemple quand monsieur Bazex a été irrité et bouleversé en disant qu'on ne peut pas

29. continuer comme ça laissez moi parler je ne vous ai pas coupé quand vous m'avez parlé et quand

30. vous m'avez cité d'assassin

D/R.Tatouage-QuestionB, 22-30.

Les émotions présentes dans le débat sont évaluées négativement en raison de leur intensité "pleine d'émotions » (exemple 2, l. 26) " trop émotionnés » (exemple 3, l. 3) et pour leur caractère agressif ,,parce qu'il attaque (exemple 2, l. 26), « désagréable » (exemple 3, l. 6). Les comportements interactifs des interlocuteurs sont souvent évalués en termes d'impolitesse. Nous en trouvons des illustrations dans l'exemple 3 ci-dessous: « les deux messieurs étaient trop pas euh trop mal éduqués » (l. 5), «ils ne s'écoutaient l'un à l'autre » (l. 6) «ils ne se respectent pas » (l. 7).

\section{Exemple 3}

B. 1. Il y a aussi cette euh (rire) cette dernière question qui parle des émotions dans la discussion et ça me

2. paraît assez complexe mais en général est-ce que à ton avis la discussion était émotionnée?

A. 3. trop émotionnée

B. 4. trop émotionnée ?

A. 5. trop oui je crois que les deux messieurs étaient très trop pas euh trop mal éduqués (rire)

6. et ils cette discussion était très désagréable pour moi ils ne s'écoutaient l'un à l'autre je crois

7. qu'ils ne se respectent pas

B. 8. Mhm

A. 9. Et il n'y avait pas l'objectivité les arguments étaient contre-arguments étaient assez ridicules ils ils ee

10. Méchants?

B. 11. mhm méchants d'accord c'est tout à fait ça à mon avis ça eee le rage ee (rire) de de de ces deux

12. messieurs ça commence au moitié il me semble à la moitié de de de l'interview parce que au début

A/B.Tatouage-QuestionA, 1-12.

Notons au passage que les arguments avancés par les intervenants sont aussi qualifiés de «méchants » et de «subjectifs »: « et il n'y avait pas l'objectivité les arguments étaient contre-arguments étaient assez ridicules» (l. 9). En s'appuyant sur les critères implicitement évoqués ici par les étudiantes, on peut supposer que pour elles l'idéal polémique est celui de l'argumentation rationnelle et objective. 
Etant donné le fait que les observations citées ci-dessus se répètent dans tous les enregistrements des apprenants, on peut penser que la facilité avec laquelle ils perçoivent et analysent les émotions au niveau interactif vient de leur caractère général (macrostructurel), de leur répétitivité et aussi de leur redondance (ils se manifestent au niveau verbal et extraverbal), ce qui favorise naturellement la focalisation de l'attention et la mémoire. Ceci n'est pas le cas des opérations de coconstruction du sens proprement dite. En effet, aucune étudiante n'évoque la stratégie de Tin-tin qui consistait à se saisir de la parole de MCC et de la transformer discursivement en rangeant ainsi les propos de MCC de son côté :

1. MCC .... mais le tatouage s'est toujours fait et dans des conditions peut-être pas très encadrées

2. dans le passé mais est-ce que nous avons comme ça des épidémies et des problèmes

3. énormes...

4. Tt n'y en a pas et aujourd'hui en plus aujourd'hui c'est tellement encadré excusez-moi de

5. de d'intervenir mais c'est tellement encadré aujourd'hui que je pense que les médecins

La matérialité langagière de cette stratégie avec la répétition $(3 \mathrm{x})$ avec insistance de « aujourd'hui » évoquant implicitement les données non actuelles reprochées au professeur Bazex dans les interventions précédentes (ici non retranscrites) et les mettant dans le même rang que le passé dont parle MCC n'est commentée dans aucune discussion des apprenants. Il en est de même avec la double répétition avec insistance de «tellement encadré » (lignes 4-5) et la troisième personne du pluriel « les médecins » pour désigner Bazex et l'exclure symboliquement du débat (délocution).

\subsection{IDENTIFICATION DE l'ETHOS}

En ce qui concerne les réponses à la question sur la stratégie rhétorique de Tin-tin, l'attention des étudiantes se focalise essentiellement sur le bien-fondé de sa comparaison entre les risques de tatouage (minimes) et la mort suite à une intervention chirurgicale (risque majeur). Comme nous l'avons déjà signalé, toutes les étudiantes l'analysent dans la perspective rationnelle (logos), en insistant sur la différence de ces situations par rapport aux risques nécessaires ou non nécessaires, comme dans le passage suivant :

\section{Exemple 4}

M. 1. En vérité je ne suis pas sûre si si on peut comparer les risques euh venant des infections euh liées

2. aux interventions chirurgicales euh et ceux liés aux tatouages, en général les interventions

3. chirugicales sont faites euh parce qu'elles doivent être faites pour des raisons de santé ou voire de la 
4. vie d'une personne par contre les tatouages sont plutot liés à la volonté de de l'avoir euh de de l'avoir

5. un tatouage donc pour moi il y a TRES grande différence entre la nécessité de mettre sa vie en

6. danger ou sa santé en danger et euh et la volonté de le faire

S. 7. oui je suis complètement d'accord avec vous euh c'est vrai bien sûr que le risque lié aux interventions

8. chirurgicales autorisées beaucoup plus importante que celui correspondant ee à se faire tatouer

9. ee mais ee le cas du tatouage dans le cas du tatu tatouage on prend ce risque délibérément, il n'y a

10. aucune contrainte au contraire euh en ce qui concerne les interventions chirurgicales euh il est vrai

11. que nous pouvons euh en conséquence de ces interventions même mourir mais ce sont euh souvent

12. les opérations qui sont qui sont obligatoires...

M. 13. ...nécessaires indispensables...

S. 14. ...oui nécessaires ils peuvent sauver notre santé ou notre vie donc cette comparaison est euh pour

15. moi cette comparaison est ee très très ee inconvéniente

M. 16. Ces deux types de risques sont sont incomparables simplement complètement incomparables. C'est

17. ça

M/S.Tatouage-QuestionA, 1-17.

Seulement la moitié des étudiantes inscrit cette comparaison dans une visée de décrédibilisation de Bazex et de la destruction de son ethos. A titre illustratif, citons l'exemple 5 :

\section{Exemple 5}

R. 1. Pour moi c'est un peu exagéré mais quand même je pense que Tin-tin l'utilise pour montrer son de

2. la manière ironique cette ce problème en fait euh bon il a dit qu'il y a plus de de hum $\mathrm{y}$ a plus de en fait

3. y a plus de cas mortels en fait dans dans l'hôpital où il y a plein de médecins etc. que euh c'est à

4. l'hôpital ça doit être le lieu sûr et bon il y a plus de cas mortels que de cas mortels à cause de

5. tatouages donc il va il utilise cette comparaison juste pour montrer que en fait monsieur Bazac cette

6. ce médecin en fait il il exagère que le problème de tatouage pour moi c'est vrai en fait, le problème de

7. tatouages n'est pas si grave que les problèmes de situations graves à l'hôpital en fait

D. 8. Oui je suis d'accord, je pense que Tin-tin est ironique mais je trouve aussi qu'il est pour les tatouages

9. dans cette façon de faire cette comparaison il soulève le savoir-faire et les compétences de médecins

10. des professionnels 
R. 11. Oui mais quand même il a essayé de souligner en fait euh sa position envers monsieur Bazex Il a

12. essayé donc cette ironie est bien fait et bien préparé je pense en fait

D/R.Tatouage-QuestionA, 1-12.

Les deux étudiantes dépassent ici la réponse à la question de "ce qui est dit" et "comment cela est dit" et vont jusqu'à la compréhension du "pourquoi" de la démarche adoptée par Tin-tin en remarquant que celui-ci l'utilise pour présenter la question de risques de tatouage sous un autre éclairage pour utiliser la terminologie de Grize (2004), (exemple 5 lignes 1-7), qu'il le fait pour signifier implicitement que le professeur Bazex " exagère le problème de tatouage " (1.6). Cette hypothèse est aussi complétée par celle sur la disqualification de l'ethos de Bazex, comme le remarque très justement $R$ dans les lignes 9-10 «dans cette façon de faire cette comparaison il soulève le savoir-faire et les compétences de médecins des professionnels ».

\subsection{IDENTIFICATION DU PATHOS}

Le peu d'attention accordée par les apprenants à l'auditoire dans le cas de l'ethos s'accompagne de leur désintérêt absolu pour le pathos: l'effet de dramatisation produit par la citation du nombre des morts suite aux infections nosocomiales en comparaison avec celles provoquées par les accidents de voiture passe inaperçu. Il en est de même des contenus implicites, basés sur les représentations partagées et soumis à la visée de persuasion. Seule la représentation de l'hôpital comme un lieu sûr est évoquée par une étudiante dans les lignes 3-4 de l'exemple $5:$ " dans l'hôpital où il y a plein de médecins etc. que euh c'est à l'hôpital ça doit être le lieu sûr ». Tout porte à croire que les étudiantes ont tendance à considérer ce débat comme une interaction entre les intervenants dans le studio et à oublier (alors qu'elles le savent très bien) que les vrais destinataires de celui-ci sont les auditeurs de radio. Encore une fois, elles se comportent comme si leur évaluation du débat devait se fonder essentiellement sur l'analyse logique des arguments.

\section{CONCLUSION}

L'examen des discussions enregistrées par les étudiantes montre que cellesci ont un bon niveau de compréhension globale. Mais il dévoile aussi un problème grave d'intégration de différents niveaux (interactif, discursif) en rapport avec la visée de persuasion (logos, pathos, ethos). En plus, leurs intuitions ne sont pas suffisamment vérifiées dans le contexte social (représen- 
tation de l'ethos p.ex.) ni dans le contexte de l'intervention analysée. Ce qui manque aussi, c'est la non prise en compte de la matérialité langagière comme source de justification, de précision et de finesse des analyses produites. On peut donc constater que dans la perspective de la création de la réception critique, les compétences des apprenants ont besoin d'être enrichies. Eu égard au développement théorique proposé dans la première partie de cet article, il semble que le travail pédagogique devrait aller dans le sens du renforcement du processus de formulation et de vérification des hypothèses en réponse aux questions du type : “pourquoi c'est dit ?", “comment c'est dit et pourquoi comme cela ?". Cela semble réalisable à travers la mise en rapport systématique des éléments langagiers (matérialité langagière) avec les visées rhétoriques propres au genre. 


\section{BIBLIOGRAPHIE}

Amossy, R. (2011/12): « Des sciences du langage aux sciences sociales : l'argumentation dans le discours $\gg$. A contrario, 16, 10-25.

AlLEN, N. (2012): Enseigner explicitement des métastratégies interprétatives en didactique de l'oral et de la lecture au deuxième cycle du secondaire avec l'album pour la jeunesse. Mémoire présenté comme exigence partielle de la maîtrise en didactique des langues. http://www.archipel.uqam.ca/5062/1/M12655.pdf (La date du dernier accès: 10.04.2014).

BrancA-RosofF, S. (2007): « Normes et genres de discours ». Langage et société, 119, 111-128.

BRETON, P. (2007): "Incompétences rhétoriques, incompétences démocratiques ». Questions de Communications, 12, 77-88.

Charaudeau, P. (2008): "Pathos et discours politique ». In : M. Rinn (coord.), Émotions et discours. L'usage des passions dans la langue. Rennes: Presses universitaires de Rennes, 49-58.

Coste, D., North, B., Sheil, J., Trim, J. (2001): Cadre européen commun de références pour les langues. Conseil de l'Europe.

Doury, M. (2003): «L'évaluation des arguments dans les discours ordinaires ». Langages et société, 105, 9-29.

Ducrot, O. (2004): « Argumentation rhétorique et argumentation linguistique ». In : M. Doury \& S. Moirand (ed.), L'argumentation aujourd'hui. Positions théoriques en confrontation. Paris : Presses Sorbonne Nouvelle, 7-34.

GÓRECKA, J., WILCZYŃSKA, W., WOJCIECHOWSKA, B. (sous presse) : "Developing second language oral competence through an integrated discursive approach: The conceptual framework of the project and the pilot study results". In : M. Pawlak \& E. Waniek-Klimczak (ed.), Theoretical, empirical and pedagogic perspectives on teaching, learning and assessing speaking skills in a second language.

GÓRECKA, J., WOJCIECHOWSKA, B. (2013): „Rozwijanie rozumienia publicystycznych audycji radiowych na poziomie zaawansowanym: specyfika etapu poprzedzającego słuchanie". Neofilolog, 40(1), 59-78.

GRIZE, J.-B. (2004): « Le point de vue de la logique naturelle : démontrer, prouver, argumenter ». In: Marianne Doury \& Sophie Moirand (ed.), L'argumentation aujourd'hui. Positions théoriques en confrontation. Paris : Presses Sorbonne Nouvelle, 35-43.

JACQUIN, J. (2011): « Le/ La polémique : une catégorie opératoire pour une analyse discursive et interactionnelle des débats publics ? » Semen, 31, 43-60.

Kerbrat-OreCChioni, C. (2005) : Discours en interaction. Paris : Armand Colin.

LAFONTAINE, L. (2004): «L'enseignement-apprentissage des genres oraux à l'école secondaire québécoise ». In : Actes du 9ème Colloque du l'AIRDF.

TindALE, CH. W. (2009): «L'argumentation rhétorique et le problème de l'auditoire complexe ». Argumentation et Analyse du Discours [en ligne] n. 2, http://aad.revues.org/493 La date du dernier accès: le 11 avril 2014.

VANDENDORPE, СH. (2001): «De la socialisation des stratégies interprétatives in Catherine Tauvernon, Comprendre et interpréter le littéraire à l'école et au-delà ». Paris INRP, 81-101.

VignauX, G. (2004): « Une approche cognitive de l'argumentation ». In : M. Doury \& S. Moirand (ed.), L'argumentation aujourd'hui. Positions théoriques en confrontation. Paris: Presses Sorbonne Nouvelle, 103-124.

Vincent, D., Bernard BARBeAu, G. (2012): «Insulte, disqualification, persuasion et tropes communicationnels : à qui l'insulte profite-t-elle? ». Argumentation et analyse du discours, 8, „Insulte, violence verbale, argumentation” la revue électronique du groupe ADAAR http://aad.revues.org/1252?lang=en la date du dernier accès: 14.03.2014. 